\title{
Database Design of Security CT Center After-sales Service System Based on MYSQL

\author{
Yongchang Ren ${ }^{\mathrm{a}}$, Yongzhe $\mathrm{Ma}^{\mathrm{b}}$
}

School of Information Science and Technology, Bohai University, Jinzhou, 121013, China

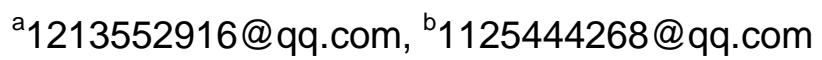

Keywords: after-sale service system; database design; conceptual structure design; logical structure design; physical structure design

\begin{abstract}
After-sales service is an important means to assure the quality of products, adopting after-sales service system can enhance service quality and work efficiency, further increase customer satisfaction. In view of the difficult problems in the development of after-sales service system, this paper uses standardized design process to design system databases normatively. First of all, a brief introduction to the adopted database and its design process; then, using entity relationship models to design the conceptual structure; after that, carrying on the logical structure design to transform the conceptual model into the specific data model supported by the database; Finally, carrying on the physical structure design for data access methods and storage structures. The design of the system database in this paper has characteristics of high security, high speed of data access and good features, which provides a powerful guarantee for the establishment of a suitable and convenient after-sales service system.
\end{abstract}

\section{Introduction}

After-sales service quality is an important guarantee of product quality, which has a direct impact on the customer satisfaction and brand influence [1], strengthening after-sales service managements has important significance for consummating the after-sales service system, standardizing the business processing and approval process, improving the quality of service. Adopting traditional management modes leads that business managements are confused, which involves many departments and personnel; the conditions that human factors lead service delay and wrong information transfer often occur. Therefore, the informational construction of after-sales service management is imminent, building security CT after-sales service system to classify and manage the business, and provide reliable and accurate information for all kinds of business of after-sales service in real time, which realizes the effective monitor of service process, guarantees within the prescribed period of time to provide clients with considerate and meticulous service, reduces the rate of customer complaints, and statistics feedback problems to make accurate analysis, provides accurate basis for repairing product defects [2].

Database design is to refer to that in a given application environment, according to the business rules to construct the best database model, so that it can store data effectively to satisfy users' various application requirements. After-sales service system needs to store a large amount of data to dispose, therefore the database design system is the most important link of the system construction, which determines the quality and running effect of the system. This article adopts the standardized design process to normatively design database.

\section{Database Design}

The quality of database design directly affects the stability and operating efficiency of the system. Therefore, the system uses the open source MYSQL database to store data, the database is currently one of the most commonly used relational database management system, which has the characteristics of easy operation, high efficiency of data processing, small volume, strong stability, fast reading and writing [3,4]. In the design process, meeting users' needs as the major premise, strictly following the design principles, such as the table name length is not more than thirty 
characters [5]; according to the three steps of database design: conceptual structure design, logical structure design, physical structure design, designing the system database normatively, so as to effectively improve the scalability, validity, integrity and availability of the data [6], which makes the database design more reasonable, efficient, high quality.

\section{Conceptual Structure Design}

Conceptual structure design is also known as conceptual design, it is the most important link in the process of database design, which is the representation process that abstract the data and users' requirements into the conceptual model which is easy to understand [7]. Through abstracting, generalizing and aggregating after-sales service business data, which gets a conceptual data model independent of a specific database management system, showed by the entity relationship diagram; this diagram is also called the E-R diagram, which provides a method of representing properties, entities and relationships used to describe the conceptual model of the real world [8]. Attributes, entities and relationships are basic elements used to constitute the E-R diagram; properties are represented by an ellipse, filled contents are on behalf of the property name, connected to the corresponding entities with undirected edges; entities are represented by a rectangular, filled contents are on behalf of the entity name; relationships are represented by an diamond, filled contents are on behalf of the contact name, respectively connected to associated entities with undirected edges, at the same time, on the side marked association types (one-to-one, one-to-many, many-to-many), among them, the one-to-one correlation is marked with $1: 1$, the one-to-many association is marked with 1 : $n$, the many-to-many association is marked with $\mathrm{m}$ : $\mathrm{n}$. The system conceptual data model design is shown in Fig. 1, because entities' attributes are too much, so they are not expressed graph.

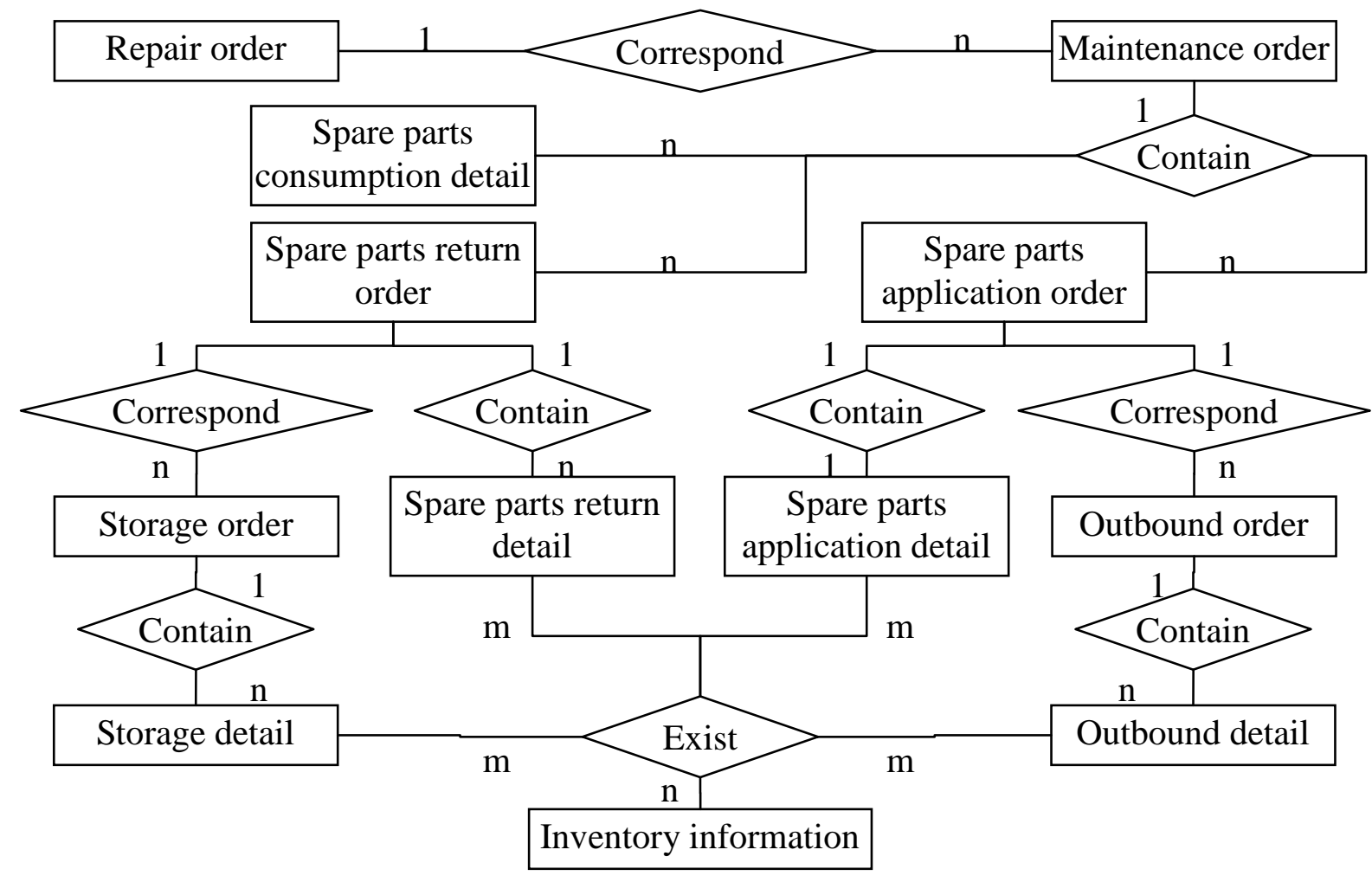

Fig. 1. After-sales service system E-R diagram

The system has 12 entities, they are "repair order, maintenance order, spare parts consumption detail, spare parts application order, spare parts application detail, spare parts return order, spare parts return detail, outbound order, outbound detail, storage order, storage detail, inventory information", the relationships between entities are as follow: 
First, there are one-to-many associations between "repair order" and "maintenance order". One or more maintenance orders can correspond to the same repair order, both are associated with repair order number as the key attribute.

Second, there are one-to-many associations between "maintenance order" and "spare parts consumption detail". One maintenance orders can contain the detailed information of one or more consumed spare parts, both are associated with maintenance order number as the key attribute.

Third, there are one-to-many associations between "maintenance order" and "spare parts application order". One maintenance order can contain the application orders of one or more types of spare parts, both are associated with maintenance order number as the key attribute.

Fourth, there are one-to-many associations between "maintenance order" and "spare parts return order". One maintenance order can contain the return orders of one or more types of spare parts, both are associated with maintenance order number as the key attribute.

Fifth, there are one-to-many associations between "spare parts application order" and "spare parts application detail". One spare parts application order owns one or more detailed information of spare parts, both are associated with spare parts application number as the key attribute.

Sixth, there are one-to-many associations between "spare parts return order" and "spare parts return detail". One spare parts return order owns one or more detailed information of spare parts, both are associated with spare parts return number as the key attribute.

Seventh, there exit one-to-many associations between "spare parts application order" and "outbound order". Applicate spare parts can be disposable or more batches of outbound, both are associated with spare parts application number as the key attribute.

Eighth, there exit one-to-many associations between "spare parts return order" and "storage order". Returned spare parts can be disposable or more batches of storage, both are associated with spare parts return number as the key attribute.

Ninth, there exit one-to-many associations between "storage order" and "storage detail". One storage order can contain one or more spare parts storage information, both are associated with storage number as the key attribute.

Tenth, there exit one-to-many associations between "outbound order" and "outbound detail". One outbound order can contain one or more spare parts outbound information, both are associated with outbound number as the key attribute.

Eleventh, there exit many-to-many associations between "spare parts application detail" and "inventory information". When one type of spare parts apply, its detailed information will exit in a variety of inventory information, one of inventory information can also storage detailed information of various types of spare parts, both are associated with inventory number as the key attribute.

Twelfth, there exit many-to-many associations between "spare parts return detail" and "inventory information". When one type of spare parts return, its detailed information will exit in a variety of inventory information, one of inventory information can also storage detailed information of various types of spare parts, both are associated with inventory number as the key attribute.

Thirteenth, there exit many-to-many associations between "outbound detail" and "inventory information". One type of spare parts can be outbound from one or more inventories, one of inventory information can also storage detailed information of various types of spare parts, both are associated with inventory number as the key attribute.

Fourteenth, there exit many-to-many associations between "storage detail" and "inventory information". One type of spare parts may be storage into many inventories, one of inventory information can also storage detailed information of various types of spare parts, both are associated with inventory number as the key attribute.

\section{Logical Structure Design}

Logical structure design is also called logical design, which is the next link for the conceptual structure design, its quality determines the success or failure of database design [9]. This stage focuses on the division of the entity and the relationship design between them, the task is to convert the concept model of the system into the data model supported by the system database, and adopt 
the design principle of the third paradigm to standardize and optimize the data structure, reduce redundancy, improve the database performance.

In the design process, transforming the conceptual model into relational model should follow certain transformation rules: when the relationships which have the same code are combined, an entity is converted into a relationship schema, entity attributes are also relational attributes, entity codes are also relational codes; a link is converted into a relational schema, its properties and its associated entity codes are both converted into relational attributes, but for different relational types, the transformation results of relational codes are different; if there is one-to-one correlation, both entity codes are transformed into the relational candidate code, otherwise, combined to relational codes [10]. According to the transformation rules, determining the main foreign key and constraint conditions to form the physical model [11], the transformation result of the system concept model are as follows. Among them, the properties marked underline are primary key words; the properties marked wavy lines are foreign key words, which are corresponding to the attribute that the primary key word of associated entities.

Repair order (repair order number, repair date, repair person, contact phone number, device number, repair location, equipment type, warranty period, order type, fault time, fault phenomenon description, received person, received time);

Maintenance order (maintenance order number, repair order number, equipment number, equipment type, repair date, repair fault description, customer name, contact person, contact phone number, maintenance fault description, optical beam time, slip ring running time, service type, the start date of engineers in transit, the end date of engineers in transit, scene service start date, scene service end date, maintenance engineers, participants, the cumulative time of break down, cumulative maintenance time, scene processing instructions);

Spare parts consumption detail (consumption number, maintenance order number, material code, material name, identification code, specifications and model, quantity);

Spare parts application order (application number, maintenance order number, the applicant, contact information, application type, application reasons, application time, received contact person, received contact address, received contact information);

Spare parts application detail (application detail number, application number, inventory number, material code, material name, specifications and model, quantity);

Spare parts return order (return number, repair order number, return type, transactor);

Spare parts return detail (return detail number, return number, inventory number, material code, material name, identification code, specifications and model, quantity);

Outbound order (outbound number, application number, type, transactor, outbound date);

Outbound detail (outbound detail number, outbound number, inventory number, material code, material name, specifications and model, quantity);

Storage order (storage number, inventory type, transactor, attachment, storage date);

Storage detail (storage detail number, storage number, return number, inventory number, material code, material name, identification code, specifications and model, quantity);

Inventory information(inventory number, spare parts type, material code, material name, identification code, specifications and model, inventory quantity).

\section{Physical Structure Design}

Physical structure design is also called physical design, which is the important work of the late database design, mainly for the design of reasonable access methods and storage structures for data. First of all, according to the designed logical structure define views, table structures, and establish index, the main foreign key, design data storage paths and storage location and so on; then, evaluate the overall performance of the structure, including time and space efficiency, and weigh user requirements and maintenance cost, and finally get a physical structure with best performance, during this period, also consider the security and integrity of the database.

After-sales service system has a large amount of data, its business association is strong. In order to save the business relationship between data and increase the data query speed, it is essential to 
create a middle table to store all the data of related tables, which maintains their relationship. And according to user requirements establish index for the field used for query conditions in the tables to improve the query efficiency; can also create a file group to storage table in different files, using disk controllers used to store files to find the required data in the table to improve query performance. In order to ensure data security and integrity, using the hot backup way to backup system data once a day; the system is equipped with at least two databases and follows the master-slave mechanism: one is a primary database, one is a secondary database, when the primary database breaks down, the system can immediately connect the secondary database, which ensures the system is normally operating.

\section{Conclusion}

After-sales service system is to make the daily after-sales service work into information, maintain basic information, record work implementation and the related business management in detail, standardize the business process flow, consummate after-sales service system, effectively improve the efficiency and quality of work, speed up the service progress, which makes the personnel division more reasonable and service more professional. Database construction has a very big influence for the information of after-sales service management. This article applies standardized methods to design system database which has characteristics of high security, high speed of data access and good features, which provides a powerful guarantee for the establishment of a suitable and convenient after-sales service system. Using the research content in this paper will play a positive promoting role in strengthening after-sales service supervision, improving economic efficiency and the quality of products for the enterprise.

\section{References}

[1] S. F. Yin, "Research of Engine Service Management Platform," Manufacturing Automation, vol. 33, no. 9, pp. 21-23, 2011.

[2] L. L. Shou, X. Y. Chen, "Design and Development of Contract Management System Based on B/S Architecture," Private Science and Technology, vol. 19, no. 3, pp. 41-42, 2013.

[3] G. Q. Jin, Y. H. Dong, "Synchronization Tuning of VLF Transmitting System," Modern Electronics Technique, vol. 34, no. 10, pp. 82-84, 2011.

[4] B. F. Yang, X. M. Zhang, "Design of Low Power Underwater Equipment Communication System," Computer Measurement and Control, vol. 20, no. 1, pp. 234-235, 2012.

[5] X. Song, X. Q. Yin, "Student Information Database Design and Development Based on B/S," Electronic Design Engineering, vol. 20, no. 5, pp. 58-60, 2012.

[6] Joo Chuan Tong, Shoba Ranganathan. Computer-Aided Vaccine Design, 2013.

[7] J. S. Li, K. Shi, "The Design of Dianchi Basin Pollution Sources Database Based on ARCSDE," Science Technology and Engineering, vol. 12, no. 8, pp. 1977-1980, 2012.

[8] G. Y. Yang, "Database Design on Contract Management Information System," Electronic Design Engineering, vol. 22, no. 5, pp. 1-3, 2014.

[9] C. M. Chen, "Logical Structure Design of The Database," Fujian Computer, vol. 28, no. 10, pp. 214-215, 2012.

[10] W. J. Cheng, "Database Design Principles and Methods of Implementation in Management Information System," Computer Knowledge and Technology, vol. 7, no. 5, pp. 994-995, 2011.

[11] H. Chen, J. M. Zhong, "Database Design and Optimization of MIS Registration System," Journal of Ningbo Polytechnic, vol. 16, no. 5, pp. 50-53, 2012. 